Revista Tecné, Episteme y Didaxis: TED. Año 2014, Número Extraordinario. ISSN Impreso: 0121-3814, ISSN web: 2323-0126

Memorias, Sexto Congreso Internacional sobre Formación de Profesores de Ciencias. 08 al 10 de octubre de 2014, Bogotá

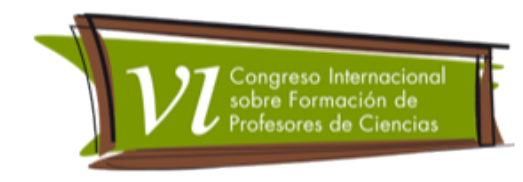

\title{
Caracterización de los Contenidos curriculares de la Química cosmética
}

Romero, Jacqueline' y Parga, Diana²

Categoría 2. Trabajo de investigación (en proceso)

\section{Resumen}

El presente escrito, presenta la problemática que actualmente existe en el país en relación con el impacto que tiene la presencia o ausencia de la asignatura Química cosmética en la formación de profesionales dedicados a la Estética Facial y Corporal. En el marco de la última innovación del contenido del syllabus de Diciembre de 2013 (proceso iniciado 15 años atrás, mediante encuestas anuales aplicadas a egresados en ejercicio), se diseñó el presente trabajo de investigación que pretende para la asignatura, entre otros: evaluar contenido temático y su secuencialidad, estudiar ubicación en el plan de estudios, establecer posibles aportes de las innovaciones del contenido del syllabus en la formación profesional y estructurar una nueva propuesta curricular.

\section{Palabras claves}

Contenido curricular, Estética Facial y Corporal, Syllabus, Química Cosmética, formación profesional.

\section{Intenciones de la investigación}

Se ha planteado como objetivo general de la investigación, evaluar en el contexto actual colombiano, el contenido curricular de la asignatura Química Cosmética, estableciendo si las innovaciones del syllabus han contribuido en la formación de profesionales idóneos en el área de la Estética Facial y Corporal, con el propósito de estructurar una propuesta macrocurricular, mesocurricular y microcurricular, la cual incluya una valoración de la ubicación de la asignatura en el plan de estudios de las instituciones que ofertan carreras de Estética Facial y Corporal en Colombia.

1 Estudiante de Maestría Docencia de la Química. Universidad Pedagógica Nacional. química_jrs@hotmail.com

2Docente Universidad Pedagógica Nacional. dparga@pedagogica.edu.co 
Revista Tecné, Episteme y Didaxis: TED. Año 2014, Número Extraordinario. ISSN Impreso: 0121-3814, ISSN web: 2323-0126

Memorias, Sexto Congreso Internacional sobre Formación de Profesores de Ciencias. 08 al 10 de octubre de 2014, Bogotá

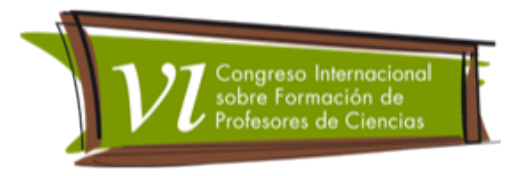

Se desea estructurar una propuesta, en el país, para ser presentada al Ministerio de Educación Nacional con la intención de definir políticas académicas en este sentido, tales como que la asignatura Química Cosmética debe ser incluida en el plan de estudios de todas las Instituciones que ofertan las Carreras de Estética Facial y Corporal en Colombia y contribuir así en la disminución de las muy graves consecuencias que se observan en algunos usuarios, las cuales son reflejo de las malas prácticas que llevan a cabo algunos profesionales que ejercen la Estética en el país.

\section{Marco teórico}

En el ámbito mundial la cátedra denominada Química Cosmética, se encuentra adscrita a los programas de instituciones de educación superior que están dedicadas a la enseñanza de la estética facial y corporal y es una de las áreas de conocimiento fundamentales que inciden en el ejercicio de la actividad de este tipo de profesional.

La Estética Facial y Corporal fue traída a Colombia hace más de 30 años. Con el transcurrir de los años, el Ministerio de Educación Nacional, profesionales de la salud y diversas entidades educativas se fueron interesando en esta área del conocimiento, lo que permitió que el estudio y la enseñanza de esta profesión fueran creciendo en el país.

Actualmente el profesional en Estética Facial y Corporal formado en Colombia incide de manera importante en el desarrollo económico e intelectual de país, brindando a la sociedad colombiana acciones importantes que mejoran la calidad de vida de las personas. A pesar de la amplia divulgación de la Estética, la tarea de un programa de alfabetización científico-tecnológico (Fourez, 1994) de orientación CTS, ha de considerar centralmente la relación de los procesos científicos y tecnológicos, y la compleja comunicación existente entre la ciencia (Química Cosmética), la tecnología (la aparatología de uso estético) y los actores (técnicas manuales) que operan en esos procesos, los cuales en su conjunto se denominan protocolos estéticos. Por ello el objetivo de la CTS es promover la participación ciudadana en la toma de decisiones concernientes al desarrollo científico y tecnológico, en alguna de las formas que se están experimentando recientemente (Martínez y Schneider, 2009; Fourez, 1994). En este sentido, se hace necesario plantear una alfabetización científica en el aula de ciencias y por lo tanto, la Química Cosmética debe considerar: 
Revista Tecné, Episteme y Didaxis: TED. Año 2014, Número Extraordinario. ISSN Impreso: 0121-3814, ISSN web: 2323-0126

Memorias, Sexto Congreso Internacional sobre Formación de Profesores de Ciencias. 08 al 10 de octubre de 2014, Bogotá

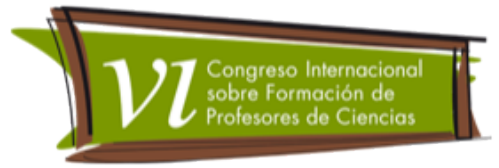

- La inclusión de contenidos actitudinales, los cuales deben ir en dos direcciones: actitudes hacia la Química Cosmética como área de conocimiento científica y actitudes de la misma hacia la sociedad (Solbes y Vilches, 2004).

- Crear currículos que vinculen la Estética y por ende la Química Cosmética, como ciencia, tecnología, sociedad y ambiente con un eje directriz en el mismo, para demostrar el impacto de las ciencias en la sociedad y llegar así a la alfabetización científica de estas dos áreas (Solbes y Vilches, 2004).

Es por esto que autores como Martínez y Parga (2013, pág. 20) plantean que "la constitución de una ciudadanía democrática con énfasis en temas polémicos relacionados con ciencia y tecnología, requiere la participación real de la ciudadanía en la toma de decisiones. Así, el futuro del conocimiento científico y tecnológico no se limita a los científicos, gobernantes, especialistas o cualquier otro actor social, siendo necesaria la conformación de una ciudadanía activa".

Asimismo, se está de acuerdo en que el profesorado de ciencias y las instituciones que las enseñan, deben descentrar su atención en la enseñanza lineal de conceptos específicos de las disciplinas que enseñan (Química, Física, Biología), ya que desconocen aspectos sociales, políticos y éticos presentes en los estudios científicos que originaron tales conceptos siendo entonces necesario incorporar la interacciones CTSA (Martínez y Parga, 2013).

En el caso de la estética es fundamental que los consumidores de tratamientos estéticos tengan una visión clara (alfabetizados científica y tecnológicamente) de los beneficios y riesgos que corren al tomar una decisión relacionada con realizarse un determinado procedimiento estético, a fin de que los ciudadanos sean capaces de ser participantes activos e inteligentes respecto a las tomas de decisión que afectan su vida (Mitcham, 1996; lbarra y López, 2001).

En Colombia, por desconocimiento de los temas que a nivel internacional estructuran la asignatura denominada Química Cosmética, en algunas instituciones dedicadas a la enseñanza de la Estética se enseñan conceptos generales de Química que no se relacionan de ninguna manera con la estructuración de los productos cosméticos, ni con la aplicación de éstos en el área de la Estética Facial y Corporal y por lo tanto, con aspectos que muestren las relaciones CTS y CTSA. El problema es aún más grave cuando se analiza el hecho 
Revista Tecné, Episteme y Didaxis: TED. Año 2014, Número Extraordinario. ISSN Impreso: 0121-3814, ISSN web: 2323-0126

Memorias, Sexto Congreso Internacional sobre Formación de Profesores de Ciencias. 08 al 10 de octubre de 2014, Bogotá

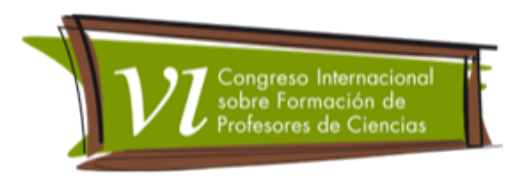

de que algunas instituciones dedicadas a la enseñanza de la Estética no incluyen en sus mallas curriculares la asignatura Química Cosmética. El enfoque poco adecuado del contenido que presenta la asignatura Química Cosmética y/o el hecho de no incluirla en las mallas curriculares, trae consecuencias muy graves que se ven reflejadas en las malas prácticas que llevan a cabo algunos profesionales que ejercen la estética en el país. Estas malas prácticas cobijan desde lesiones muy leves, pasando por lesiones muy graves y hasta la muerte de muchos usuarios de los servicios que son ofrecidos en el área estética.

\section{Metodología}

El estudio que se realizará es de caso, y será llevado a cabo desde una perspectiva cualitativa. Se constituye en una estrategia encaminada a la toma de decisiones. En esta investigación el estudio de caso es de tipo comunitario y particular porque pretende generar descubrimientos y estudiar el impacto que puede tener la caracterización de los contenidos curriculares de la asignatura Química Cosmética en relación con la formación de profesionales que ejercen la Estética Facial y Corporal en Colombia en el contexto actual. La investigación se considera descriptiva porque pretende estructurar una fecunda e intensa descripción del fenómeno estudiado; por último, la investigación se considera inductiva, puesto que a partir del análisis de los datos obtenidos llegará a conceptos relacionados con la innovación consistente en la renovación macro, meso y microcurricular de la asignatura Química Cosmética (Arnal, Del Rincón, Latorre, 1992; Bell, 2005). Este trabajo se suscribe dentro de la línea de investigación didáctica de los contenidos curriculares del grupo Alternaciencias de la UPN.

Son participantes de la investigación seis instituciones educativas colombianas que se caracterizan porque dos de ellas cuentan en su malla curricular con la asignatura Química Cosmética, otras dos no tienen dentro del plan de estudios la asignatura y las otras dos han incorporado desde hace varios años la implementación anual de las innovaciones del syllabus.

La información se recolectará mediante técnicas tales como entrevistas, encuestas (validadas por pares externos), grupos de discusión y análisis documental.

El trabajo se desarrollará en tres fases: fase de diagnóstico, fase de diseño curricular y la fase de evaluación de la propuesta. 
Revista Tecné, Episteme y Didaxis: TED. Año 2014, Número Extraordinario. ISSN Impreso: 0121-3814, ISSN web: 2323-0126

Memorias, Sexto Congreso Internacional sobre Formación de Profesores de Ciencias. 08 al 10 de octubre de 2014, Bogotá

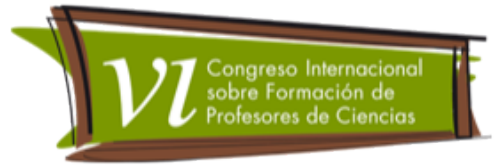

En la fase de diagnóstico se tomará como punto de partida el análisis documental de las seis instituciones dedicadas a la enseñanza de la Estética Facial y Corporal, en cuanto se refiere al contenido temático del plan de estudios y al contenido del currículo de la asignatura Química Cosmética.

Con la implementación de las técnicas de recolección, en especial las entrevistas, encuestas y grupos de discusión, entre egresados, estudiantes y docentes de las seis instituciones para las cuales se llevó a cabo el análisis documental, se pretenden diagnosticar las siguientes categorías y subcategorías de análisis:

Tabla 1. Categorías y subcategorías de análisis para el currículo de la Química Cosmética

\begin{tabular}{|c|c|c|c|c|}
\hline Categorías & \multicolumn{4}{|c|}{ Sucategorías } \\
\hline \multirow{2}{*}{ Contenidos } & $\begin{array}{l}\text { Qué y cuándo } \\
\text { enseñar }\end{array}$ & $\begin{array}{l}\text { Por qué o para } \\
\text { qué enseñar }\end{array}$ & A quién enseñar & Cómo enseñar \\
\hline & Con qué enseñar & Dónde enseñar & Qué, cuándo y cc & o evaluar \\
\hline \multirow{2}{*}{ Social } & \multicolumn{4}{|c|}{ Función social de la Química Cosmética } \\
\hline & \multicolumn{4}{|c|}{ Enfoque CTS de la Química Cosmética } \\
\hline \multirow{2}{*}{$\begin{array}{c}\text { Histórico - } \\
\text { Epistemológica }\end{array}$} & \multicolumn{4}{|c|}{ Naturaleza de la Química Cosmética como ciencia } \\
\hline & \multicolumn{4}{|c|}{ Imagen de la Química Cosmética y de su enseñanza } \\
\hline Didáctica & \multicolumn{4}{|c|}{$\begin{array}{l}\text { Explicar si la selección y secuenciación de los contenidos está de acuerdo con el } \\
\text { nivel de desarrollo de los estudiantes y de sus intereses }\end{array}$} \\
\hline
\end{tabular}

Adicionalmente se diagnosticarán los siguientes aspectos:

a) Tiempo de ejercicio profesional del egresado en el área de la Estética Facial y Corporal.

b) Si el egresado recibió o no capacitación en Química Cosmética.

c) Si el egresado recibió instrucción en la asignatura Química Cosmética, sobre qué temas específicos fue orientado.

d) En qué semestre académico se debe iniciar la enseñanza de la asignatura Química Cosmética.

e) En cuántos semestres académicos se debe abarcar la enseñanza de la asignatura Química Cosmética, según el punto de vista del profesional.

f) Si desde el punto de vista del egresado, las innovaciones del syllabus han contribuido en la formación de profesionales idóneos en el área de la Estética Facial y Corporal. Esto con el propósito de contar con antecedentes que sustenten la necesidad de una alfabetización científica en la sociedad y que se ha constituido como primordial para mejorar la educación (Gallego Torres y 
Revista Tecné, Episteme y Didaxis: TED. Año 2014, Número Extraordinario. ISSN Impreso: 0121-3814, ISSN web: 2323-0126

Memorias, Sexto Congreso Internacional sobre Formación de Profesores de Ciencias. 08 al 10 de octubre de 2014, Bogotá

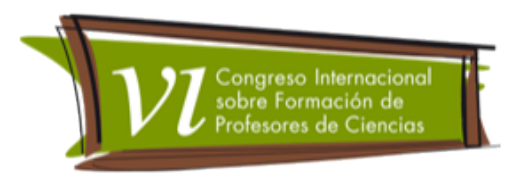

Gallego Badillo, 2009) y la calidad de vida; alfabetización que debe verse reflejada en la toma de decisiones asociadas a aspectos de la salud que involucren tratamientos 0 procedimientos estéticos desarrollados por profesionales idóneos que conozcan los impactos de los productos químicos y técnicos especializados.

En la fase de diseño curricular, a partir de la triangulación de las entrevistas, encuestas y grupos de discusión, se procederá a:

- Caracterizar el contenido curricular de la Química Cosmética presente en la actualidad, en la formación de profesionales idóneos en el área de la Estética Facial y corporal.

- Determinar si la constante modificación microcurricular de la asignatura Química Cosmética que se ha llevado a cabo en un período de 15 años, ha contribuido en el ámbito nacional en la formación de profesionales idóneos en el área de la Estética Facial y Corporal.

- Establecer cuáles deben ser las características que deben tener los contenidos curriculares de la química cosmética en la formación de profesionales idóneos en el área de la Estética Facial y corporal.

Durante la fase de evaluación de la propuesta, y a partir de la tabulación de encuestas que serán aplicadas a los egresados de las seis instituciones participantes de la investigación, se establecerán los siguientes aspectos:

a. Si están de acuerdo con el contenido de la asignatura y su secuencialidad.

b. Si es diferente al contenido microcurricular que ellos estudiaron.

c. Si la creen suficiente para mejorar la calidad académica de los estudiantes de pregrado.

d. Si propende hacia un ejercicio más idóneo de la Carrera de Estética Facial y Corporal en Colombia.

e. Si están de acuerdo con el semestre académico en el cual se debe iniciar la enseñanza de la asignatura.

f. Si están de acuerdo con el semestre académico en el cual se debe finalizar la enseñanza de la asignatura.

g. Si es pertinente que la asignatura Química Cosmética sea incluida en el plan de estudios de todas las Instituciones que ofertan las Carreras de Estética Facial y Corporal en Colombia. 
Revista Tecné, Episteme y Didaxis: TED. Año 2014, Número Extraordinario. ISSN Impreso: 0121-3814, ISSN web: 2323-0126

Memorias, Sexto Congreso Internacional sobre Formación de Profesores de Ciencias. 08 al 10 de octubre de 2014, Bogotá

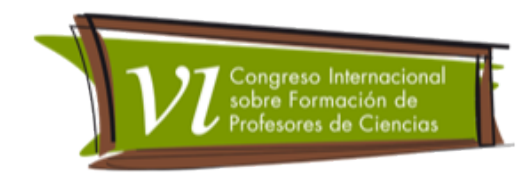

\section{Resultados esperados}

Con la presente investigación se esperan obtener los siguientes resultados:

- Estructurar una nueva propuesta de contenido macro, meso y microcurricular de la asignatura Química Cosmética.

- Estimar en qué semestre académico se debe comenzar la enseñanza de la asignatura Química Cosmética.

- Evaluar durante cuántos semestres académicos se debe enseñar la asignatura Química Cosmética.

- Contar con antecedentes que sustenten el hecho que la alfabetización científica en la sociedad Colombiana si se ha constituido en una necesidad primordial para mejorar el contenido curricular de la asignatura Química Cosmética.

- Argumentar desde los resultados obtenidos que es urgente incluir en el plan de estudios de todas las Instituciones que ofertan las Carreras de Estética Facial y Corporal en Colombia, la asignatura Química Cosmética.

\section{Referencias bibliográficas}

Arnal, J., Del Rincón, D. y La Torre, A. (1992). Investigación educativa, fundamentos y metodología. Barcelona: Editorial Labor.

Bell, J. (2da. Ed). (2005). Cómo hacer tu primer trabajo de investigación. Guía para investigadores en educación y ciencias sociales. Barcelona: Gedisa Editorial.

Fourez, G. (1994). Alfabetización científica y tecnológica. Recuperado de:http://books.google.com.co/books?hl=es\&lr=\&id=_t4hCUOYG7gC\&oi=fn d\&pg =PA3\&dq=fourez+alfabetizaci\%C3\%B3n+cient\%C3\%ADfica+tecnol\%C3 \%B3gica\&ots=0iXSjOnFtC\&sig=1 PcbPmo2r2yHDhrjaWc0Y9mGD3M\#v=onepa ge\&q=fourez\%20alfabetizaci\%C3\%B3n\%20cient\%C3\%ADfica\%20tecnol\%C3\% B3gica\&f=false

Gallego, Torres, A. P. y Gallego Badillo, R. (2009). El cambio climático. La catástrofe que nos acecha. Bogotá: Ossal. 
Revista Tecné, Episteme y Didaxis: TED. Año 2014, Número Extraordinario. ISSN Impreso: 0121-3814, ISSN web: 2323-0126

Memorias, Sexto Congreso Internacional sobre Formación de Profesores de Ciencias. 08 al 10 de octubre de 2014, Bogotá

Ibarra, A. y López, J. (2001). Desafíos y tensiones actuales en ciencia, tecnologíay sociedad. Madrid: Editorial Biblioteca Nueva, S. L.

Martínez, M. S. y Schneider F. (2009). Los enfoques Cts y Ctsa en la enseñanza de las ciencias. Biológica, No. 13. Recuperado de: schneiderf@editorialestrada.com.ar

Martínez, L. y Parga, L. (2013).Discurso ético y ambiental sobre cuestiones sociocientíficas: aportes para la formación del profesorado. Bogotá: Universidad Pedagógica Nacional.

Mitcham, C. (1996). Los estudios de Ciencia, Tecnología y Sociedad. Una introducción conceptual. En: Para comprender Ciencia, Tecnología y Sociedad. Lizarra: Verbo Divino pág. 9-16.

Solbes, J. y Vilches, A. (2004). Papel de las relaciones entre ciencia, tecnología, sociedad y ambiente en la formación ciudadana. Enseñanza de las ciencias, 22(3), pág. 337-348. Recuperado el 10 de Agosto de 2013 de: http://ddd.uab.es/pub/edlc/02124521v22n3p337.pdf 